\title{
New results from isochronous mass measurements of neutron-rich uranium fission fragments with the FRS-ESR-facility at GSI
}

\author{
${ }^{1}$ R. Knöbel, ${ }^{2, *, * *}$ M. Diwisch, ${ }^{1,2}$ H. Geissel, ${ }^{1}$ Yu. A. Litvinov, ${ }^{3}$ Z. Patyk, ${ }^{1,2}$ W. R. Plaß, ${ }^{1,2}$ C. Scheidenberger, \\ ${ }^{4,1}$ B. Sun, ${ }^{1}$ H. Weick, ${ }^{1}$ F. Bosch, ${ }^{1}$ D. Boutin, ${ }^{1,2}$ L. Chen, ${ }^{1}$ C. Dimopoulou, ${ }^{1}$ A. Dolinskii, \\ ${ }^{1}$ B. Franczak, ${ }^{1}$ B. Franzke, ${ }^{5}$ M. Hausmann, ${ }^{1}$ C. Kozhuharov, ${ }^{1}$ J. Kurcewicz, ${ }^{1}$ S. A. Litvinov, \\ ${ }^{1}$ M. Matoš, ${ }^{1}$ M. Mazzocco, ${ }^{1}$ G. Münzenberg, ${ }^{6}$ S. Nakajima, ${ }^{1}$ C. Nociforo, ${ }^{1}$ F. Nolden, ${ }^{7}$ T. Ohtsubo, \\ ${ }^{8}$ A. Ozawa, ${ }^{1}$ J. Stadlmann, ${ }^{1}$ M. Steck, ${ }^{6}$ T. Suzuki, ${ }^{9}$ P. M. Walker, ${ }^{1}$ M. Winkler, and ${ }^{6}$ T. Yamaguchi \\ ${ }^{1}$ GSI Helmholtzzentrum für Schwerionenforschung GmbH, 64291 Darmstadt, Germany \\ ${ }^{2}$ II. Physikalisches Institut, Justus-Liebig-Universität Gießen, 35392 Gießen, Germany \\ ${ }^{3}$ National Centre for Nuclear Research - NCBJ Swierk, Hoża 69, 00-681 Warszawa, Poland \\ ${ }^{4}$ School of Physics and Nuclear Energy Engineering, Beihang University, Beijing 100191, China \\ ${ }^{5}$ Michigan State University, East Lansing, Michigan 48824, USA \\ ${ }^{6}$ Department of Physics, Saitama University, Saitama 338-8570, Japan \\ ${ }^{7}$ Department of Physics, Niigata University, Niigata 950-2181, Japan \\ ${ }^{8}$ Institute of Physics, University of Tsukuba, Ibaraki 305-8571, Japan \\ ${ }^{9}$ Department of Physics, University of Surrey, Guildford, GU2 7XH, United Kingdom \\ *Part of Doctoral Thesis, Justus-Liebig-Universität Gießen, 2015 and \\ ${ }^{* *}$ Corresponding author
}

(Dated: January 29, 2016)

\begin{abstract}
Masses of uranium fission fragments have been measured with the FRS-ESR facility at GSI. A $410-415 \mathrm{MeV} / \mathrm{u}{ }^{238} \mathrm{U}$ projectile beam was fast extracted from the synchrotron SIS-18 with an average intensity of $10^{9} /$ spill. The projectiles were focused on a $1 \mathrm{~g} / \mathrm{cm}^{2}$ beryllium target at the entrance of the in-flight separator FRS to create neutron-rich isotopes via abrasion-fission. The fission fragments were spatially separated with the FRS and injected into the isochronous storage ring ESR for fast mass measurements without applying cooling. The Isochronous Mass Spectrometry (IMS) was performed under two different experimental conditions, with and without $\mathrm{B} \rho$-tagging at the high-resolution dispersive central focal plane of the FRS. A new method of data analysis has provided experimental mass values for 25 different neutron-rich isotopes for the first time and 6 masses with improved uncertainties. The results were obtained by the use of a correlation matrix for the combined data set from both experiments. The new masses were obtained for nuclides in the element range from Ge to Ce. The applied analysis has given access even to rare isotopes detected with an intensity of a few atoms per week. The novel data analysis and systematic error determination are described and the results are compared with extrapolations of experimental values and theoretical models.
\end{abstract}

\section{INTRODUCTION}

The mass of an atomic nucleus is a fundamental property, representing the total binding energy of the nucleons. The total binding energy is the sum of all interactions of the constituents dominated by the nuclear force. From this statement it is evident that accurate mass values reflect also details of the evolution of nuclear structure and stability as well as the energy levels and spatial distributions of the bound nucleons. Therefore, accurate mass values are a crucial test for the predictive power of nuclear models [1]. Reliable mass values are needed as input for reaction studies where the Q-values or the nucleon separation energies play a crucial role, e.g., for the understanding of the abundance of nuclei produced in stellar matter [2].

The advent and application of radioactive nuclear beam facilities [5] and novel mass spectrometers [6] have dramatically enlarged the number of known isotopes $[7,8]$ with unusual proton-to-neutron ratios and thus the nuclear properties can be studied at the outskirts of the chart of nuclides. From systematic mass measurements it became evident that the nuclear shell structure can change towards the driplines. Shell quenching, complete disappearance, or even new magic numbers have been theoretically predicted $[9,10]$ and observed in experiments [11-13]. Mass measurements of exotic nuclei are a great experimental challenge because the isotopes of interest are short-lived and are produced in nuclear reactions with very low cross sections. Furthermore, they can only be investigated if they are efficiently separated from the non-reacted primary beam and all abundant contaminants. Exotic nuclei are excited due to their creation process in reactions, therefore, they may often enter the mass spectrometers in long-lived excited states. It means, the ions have different masses depending on their excitation level. It is the goal of accurate mass measurements to extract the ground-state mass and resolve the different states. The latter task is a great experimental challenge and depends mainly on the excitation energy and the population of the different states. Experimentalists cope with this challenge by new developments and techniques yielding higher mass resolving power. However, the de- 


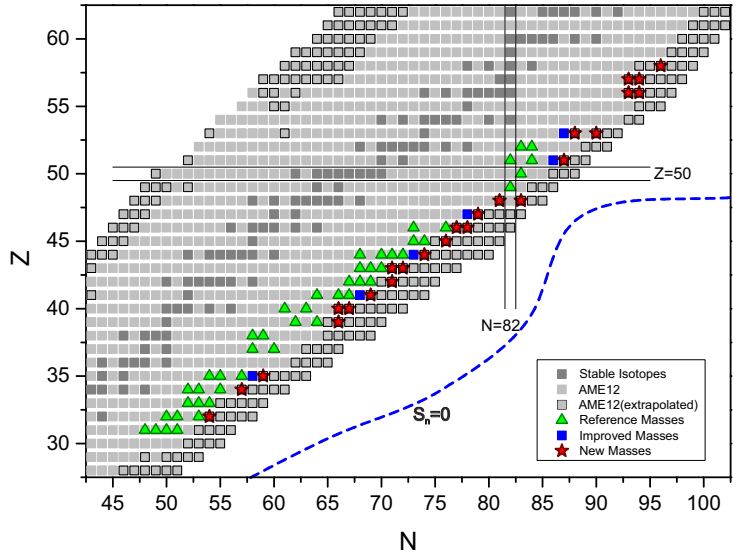

FIG. 1. Nuclides covered in the present IMS experiments with the FRS-ESR facilities. The masses measured for the first time are indicated by stars. The reference masses used in the analysis and the neutron dripline [3] are shown as well. The new IMS mass measurements of neutron-rich Cd isotopes are indicated but have been separately published [4].

vices with the highest resolution are critically prone to small drifts and changes of the experimental conditions. The situation is even more severe if only a few ions are recorded over a week or longer. This challenge characterises the present experiments which represent a pilot investigation for the limitation of future experiments.

The highest mass accuracy and resolving power has been reached with frequency and revolution time measurements in ion traps or storage rings $[6,14,15]$. Many of the described challenges with exotic nuclei can be solved by frequently recorded calibration masses or, even better, when the reference masses are simultaneously measured in the same spectrum together with the ions with unknown experimental masses.

In this contribution, we present new results from isochronous mass measurements with the FRS-ESR facility at GSI. The Isochronous Mass Spectrometry (IMS) [16] was performed under two different experimental conditions, with and without $\mathrm{B} \rho$-tagging [17] at the dispersive central focal plane of the FRS [18]. A goal was to cover in both experiments the same mass-over-charge $(\mathrm{m} / \mathrm{q})$ range to investigate the influence of the additional $\mathrm{B} \rho$ determination. It was known that in addition to the revolution time also the magnetic rigidity or equivalently the velocity measurement is mandatory for unambiguous particle identification and that corrections of the deviations from isochronicity in IMS measurements of a broader $\mathrm{m} / \mathrm{q}$-range are required [17]. The data of the two different experiments were combined and analysed together with a new correlation matrix method based on the analysis which was first applied in Schottky Mass Measurements with stored electron-cooled ions [19]. The experiments and the analysis are described in detail in this paper and the prospects and limitations of IMS are discussed. The masses covered in the present experiments are shown in the chart of nuclides in figure 1.

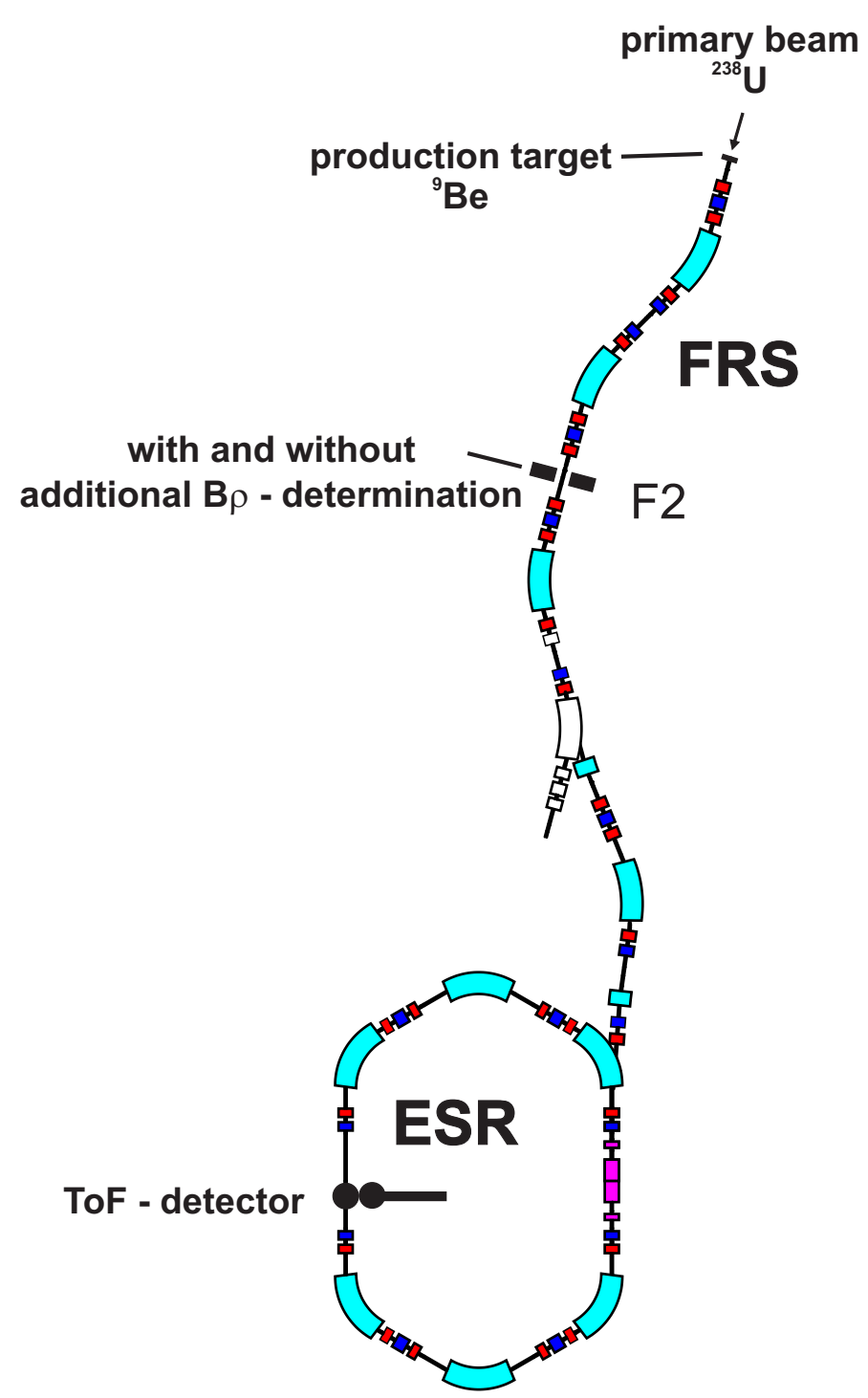

FIG. 2. The in-flight fragment separator FRS combined with the storage-cooler ring ESR for IMS experiments. The position of the production target, the central focal plane F2 of the FRS and the magnetic elements of the two facilities are depicted. Magnets which are not used are indicated in white color. The magnetic rigidity $(\mathrm{B} \rho)$ of the injected fragments was defined with mechanical slits placed at the central dispersive focal plane F2 of the FRS. The first experiment was performed with no slits, the second one had applied slits with an opening of $\pm 0.5 \mathrm{~mm}$. The position of the Time-of-Flight (ToF) detector is indicated in the ESR lattice. 


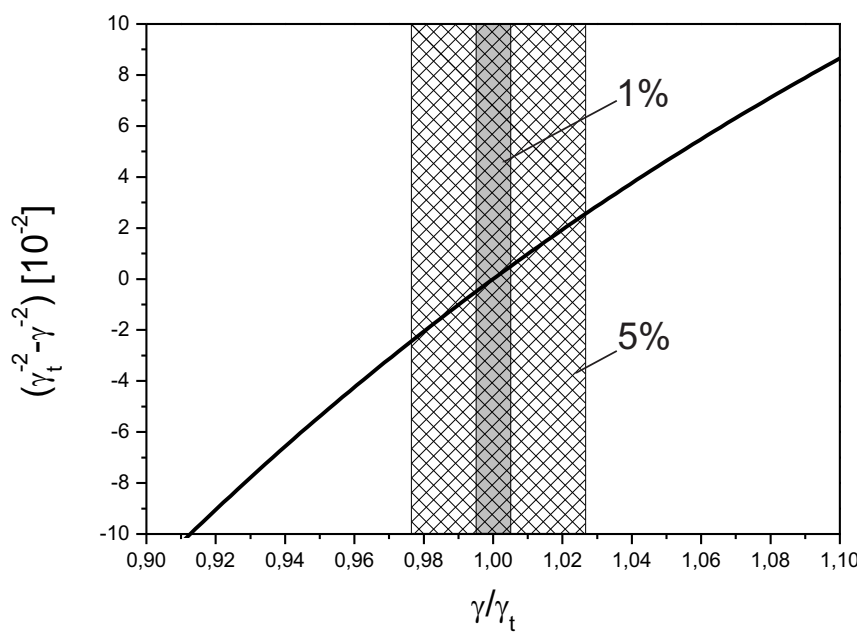

FIG. 3. Calculated correction factor $\left(1 / \gamma_{t}^{2}-1 / \gamma^{2}\right)$ dependent on the mean velocity deviation between isochronous $\left(\gamma_{t}\right)$ and non-isochronous particles $(\gamma) . \gamma$ and $\gamma_{t}$ are the relativistic Lorentz-factor and the isochronous ring parameter corresponding to the so-called transition energy. In the present calculation $\gamma_{t}$ is 1.41. Note that in the experiment $\gamma_{t}$ is not constant over the full acceptance of the ESR [16]. The windows for a m/q range of $1 \%$ and $5 \%$ are illustrated in the figure.

\section{EXPERIMENTS}

Neutron-rich exotic nuclei were produced by abrasionfission reactions of (410-415) $\mathrm{MeV} / \mathrm{u}^{238} \mathrm{U}$ ions in a $1 \mathrm{~g} / \mathrm{cm}^{2}$ Be target placed at the entrance of the FRS

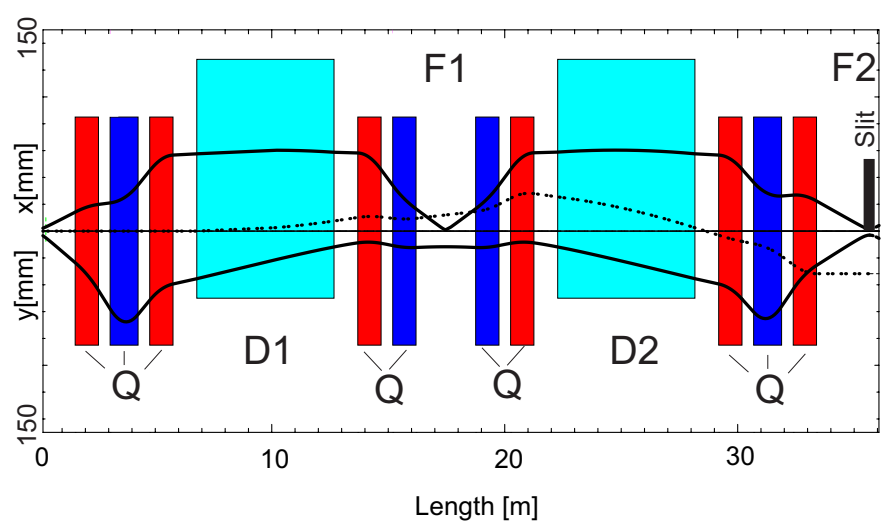

FIG. 4. Ion-optical calculations [20] of the envelopes in horizontal (x) and vertical direction (y) from the production target of the FRS up to the central focal plane. The emittance in both directions is $20 \pi \mathrm{mm}$ mrad in this calculation. The dashed line represents the dispersion coefficient for a momentum deviation of $0.5 \%$. The 30-degree dipole (D) and quadrupole (Q) magnets are drawn in the optical path. Mechanical slits with an opening of $\pm 0.5 \mathrm{~mm}$ defined the $\mathrm{B} \rho$ for the fragments injected into the $\mathrm{ESR}$. The $\mathrm{B} \rho$ resolving power of the first two ion-optical stages of the FRS are added.

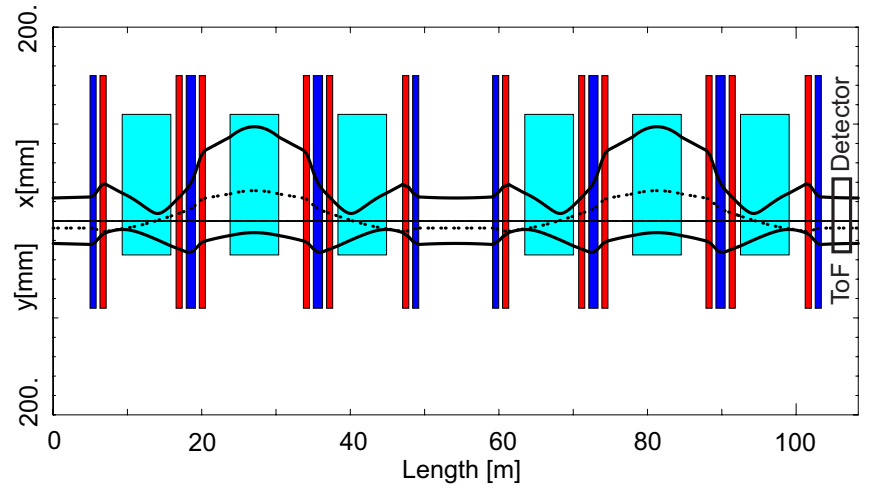

FIG. 5. Ion-optical calculations [20] of the envelopes in horizontal (x) and vertical direction (y) for the isochronous mode of the ESR. The emittance in both directions was $20 \pi \mathrm{mm}$ mrad in this calculation. The dashed line represents the dispersion line for a momentum deviation of $0.2 \%$. The dipole and quadrupole magnets are drawn in the optical path. The position of the Time-of-Flight (ToF) detector is also indicated in the ESR lattice.

[18]. The experimental setup with the FRS and ESR is schematically shown in figure 2. The ion-optical system with the dipole and multipole magnets is illustrated with the main components of the IMS experiments presented in this contribution. The kinetic energy of the incident projectile beam has been adjusted to match the magnetic rigidity $(\mathrm{B} \rho)$ of the selected pilot fission fragment, e.g., fully ionized ${ }^{133} \mathrm{Sn}$ and ${ }^{135} \mathrm{Sn}$ ions were chosen to determine the magnetic fields of the FRS and the isochronous ESR [21]. The synchrotron SIS-18 [22] provided an average intensity of the primary beam of about $10^{9}$ ions per spill. The spill lengths of the fast extracted ions from SIS-18 had a width of $(0.2-0.5) \mu \mathrm{s}$. This condition excludes the application of particle detectors recording single ions via event-by-event coincidence measurements, especially also due to low transmission of the injection channel of the ESR which is a few percent relative to the phase-space provided by FRS. The kinetic energy of the primary beam was chosen such that the mean velocity of ${ }^{133} \mathrm{Sn}^{50+}$ and ${ }^{135} \mathrm{Sn}^{50+}$ fragments corresponded to the relativistic Lorentz factor of $\gamma=1.41$, which matches the transition energy $\left(\gamma_{t}\right)$ of the ESR. The separation mode of the FRS without energy degraders was enabled by the large mean velocity difference of the projectile fragments and fission products and the restricted angular acceptance of the FRS. Practically this means that a suitable $B \rho$-selection with the FRS can provide fission-fragment beams without significant contributions of projectile fragments. Since no energy degrader was used in the present experiments, all fission products with the same magnetic rigidity were transported through the FRS and were injected into the storage ring. The transmission of the ion-optical systems of FRS and ESR was optimized with the primary beam centred at the identical mean mag- 
netic rigidity as for the chosen pilot fragments. Unfortunately, the injection acceptance of the ESR was about one order of magnitude smaller than the one of FRS. Previously, we have demonstrated that for IMS experiments in addition to the revolution time of the stored ions the magnetic rigidity or velocity measurement is required [17]. The reason is that the isochronicity condition is strictly realized only for one single mass-over-charge $(\mathrm{m} / \mathrm{q})$ value for a fixed ion-optical isochronous field setting of the ESR. The illustration of the relevance of this additional measurement for IMS experiments can be easily demonstrated with the first-order formula, relating the revolution time $(T)$ directly to the mass-to-charge ratio $(\mathrm{m} / \mathrm{q})$ of the stored and measured particle:

$$
\frac{d T}{T}=\frac{1}{\gamma^{2}} \frac{d(m / q)}{m / q}+\left(\frac{1}{\gamma_{t}^{2}}-\frac{1}{\gamma^{2}}\right) \frac{d(B \rho)}{B \rho},
$$

with $\gamma_{t}$ being an ion-optical parameter of the storage ring (transition energy), $\gamma=\left(1-(v / c)^{2}\right)^{-1 / 2}$ the Lorentz factor of the revolving particle, $v$ and $c$ the velocity of the particle and the speed of light in vacuum, respectively. In the isochronous mode of the storage ring $\gamma_{t}$ is equal to the Lorentz factor $\gamma$ for one single $m / q$ value. Only for these ions the measurement of the revolution time $T$ unambiguously determines the $m / q$ ratio. For other nuclides, the shift of the mean velocity and distribution width do not cancel and have to be taken into account. The equation 1 clearly demonstrates that it is necessary to measure the velocity and magnetic rigidity of each nuclide in addition to its revolution time, in order to achieve accurate mass values and high mass resolution. The additional quantity which has to be measured is: $\left(\frac{1}{\gamma_{t}^{2}}-\frac{1}{\gamma^{2}}\right)$. Its magnitude is in the few percent range and strongly depends on how much the velocity of a stored ion differs from the transition energy of the storage ring. The impact of the relation is presented in figure 3. Two windows for an $\mathrm{m} / \mathrm{q}$ range of $1 \%$ and $5 \%$ are illustrated in the figure.

Experimentally it is clear that these additional measurements have to be performed to achieve accurate mass values in IMS experiments with the ESR. Particle detectors operated in coincidence at the FRS cannot be used for this task, because of the spill structure of fast extracted beams from the heavy-ion synchrotron (SIS18), see above. On the other hand, the operation with fast extracted beams is necessary for the injection scenario into the storage orbit of the ESR by exciting a fast kicker magnet. Therefore, we implemented mechanical variable slits with an opening of $\pm 0.5 \mathrm{~mm}$ placed at the central dispersive focal plane of the FRS [23]. The opening and the central position of the slits were variable. The FRS is used as a high-resolution two-stage spectrometer [24]. The $\mathrm{B} \rho$ resolving power of the first two ion-optical stages of the FRS are added. The resolution of the lateral dispersive system is characterized by the ratio of the dispersion coefficient and the magnification, the image condition and the spot size at the production target, see figure 4 . In principle, a $\mathrm{B} \rho$ determination in addition to the revolution-time measurement would cause less transmission losses if it could be performed inside the storage ring. However, this was not possible, simply because the required $\mathrm{B} \rho$ resolving power could not be achieved by lateral dispersive measurements in the storage ring. The lateral resolving power of the FRS in the central dispersive focal plane is more than a factor of 10 superior for the same emittance compared to the ESR in the isochronous storage mode. This is based on ion-optical calculations [20] with the optical elements of the FRS from target to the second focal plane, see figure 4 , and from the corresponding calculations of the isochronous ESR, see figure 5. The superior lateral resolving power of the FRS is clearly illustrated by the image sizes at the focal planes in the dispersive direction. Ion-optically this is achieved by the condition that the image size at the focal plane should not depend on the angular distribution at the entrance of the system. With mechanical slits of an opening of $\pm 0.5 \mathrm{~mm}$ at $\mathrm{F} 2$, one can achieve a $\mathrm{B} \rho$ definition for the injected fragments of about $2.5 \cdot 10^{-4}$ if the slits are placed at the optical image position in the central focal plane. The slits had a length of $20 \mathrm{~cm}$ along the beam axis which reduced the transmitted angular range in addition. In the experiment the effective $\mathrm{B} \rho$ acceptance of the ESR was even smaller and amounted to $1.5 \cdot 10^{-4}$.

One goal of these pilot experiments was to explore the conditions and requirements for future IMS experiments. The present IMS measurements have been performed with and without $\mathrm{B} \rho$-tagging for the same settings of the magnetic fields of the FRS and ESR. The revolution times of the circulating ions in the storage ring were measured with a time-of-flight ( $\mathrm{ToF}$ ) detector equipped with a thin carbon foil and two micro-channel-plate (MCP) branches [25] placed in a homogeneous magnetic dipole field of about $8.4 \mathrm{mT}$ and an electric field of $154 \mathrm{~V} / \mathrm{mm}$. The created secondary electrons released from the carbon foil were isochronously deflected onto the MCPs to generate timing signals at each turn. The signals were recorded with digital oscilloscopes (Tektronix TDS $6154 \mathrm{C}, 40 \mathrm{GS} / \mathrm{s}, 15 \mathrm{GHz}$; LeCroy LC584AM, $4 \mathrm{GS} / \mathrm{s}$, $1 \mathrm{GHz})$. The rate of fragments stored in the ESR was about 1-10 per measurement cycle, which was chosen to be $20 \mathrm{~s}$. The experiments were performed over a period of about 2 weeks running time. The kinetic energy of the selected fragments was in the range of $(370-480) \mathrm{MeV} / \mathrm{u}$. At these velocities the fission fragments emerged from the production target mainly as bare ions. This condition greatly facilitated the particle identification in the ToF spectra. The recording time of (0.8-1) ms was chosen to measure up to about 2000 revolutions in the ESR. The revolution times, of about $500 \mathrm{~ns}$, for the stored ions were obtained from the measured time stamps for each stored ion and were accumulated in a revolution time spectrum 
(ToF spectrum)

\section{DATA ANALYSIS}

The mass of the stored fragment was determined by recording the revolution time $(T)$ with the ToF detector. The principle of the measurement can be understood by the equation (1). Primarily, the revolution time had to be determined from the detector signals recorded over several hundred turns. After the pattern recognition, i.e., the search for the characteristic time intervals for a specific circulating ion with a selected $\mathrm{m} / \mathrm{q}$ value, the averaged revolution time for a recorded ion was determined by a third order polynomial fit. Each ion reached an individual maximum number of turns $\left(N_{\max }\right)$, which is determined by the initial coordinates (emittance), the atomic collisions in the detector foil, rest gas and finally the detection efficiency. Therefore, the final value of $T$, which enters in the analysed time spectrum, was individually derived from a fitted curve at the revolution number of $N_{\max } / 2$.

The time resolution and thus the mass resolution depends on several experimental conditions. The main contributions are the isochronous condition of the ESR, the timing performance of the detector, the accuracy of the determination of the time stamps (software constant fraction method) and the uncertainty of the mean revolution time. The accuracy of the mean revolution time strongly depends on the achieved number of turns [27]. For selected ions which all had reached more than 250 turns the non-perfect isochronicity of the ring especially dominated the time (mass) resolution for ions with $\mathrm{m} / \mathrm{q}$ ratios quite different from the pilot beam. In figure 6 the experimentally achieved time (mass) resolution is shown for different $\mathrm{m} / \mathrm{q}$ values. It is observed that the time spread and thus the mass $(\Delta m)$ resolution almost linearly depends on the $\mathrm{m} / \mathrm{q}$ difference to the pilot beam. In this case, the selected pilot beam had an $\mathrm{m} / \mathrm{q}$ value of 2.65 corresponding to ${ }^{133} \mathrm{Sn}^{50+}$ ions. The measured time resolution was recorded under the full acceptance of the ESR and with $\mathrm{B} \rho$-tagging. The measured $\mathrm{B} \rho$ spread of the circulating fragments was with $\mathrm{B} \rho$-tagging $\frac{\Delta B \rho}{B \rho}=1.5 \cdot 10^{-4}$ and with open slits at the central focal plane (F2) of the FRS $\frac{\Delta B \rho}{B \rho}$ $=5 \cdot 10^{-4}$. The corresponding values determined for the fragments at F2 and from measurements at the injection septum of the ESR were roughly a factor of two larger. This means that stored ions circulating at least 250 turns in the ESR had experienced a further reduction of emittance in the ESR and also a loss of stored ions. In figure 7 we present a part of a typical ToF spectrum under the condition with $\mathrm{B} \rho$-tagging and without. Very clearly it can be observed that the resolving power improved by a factor 2-4 with additional $\mathrm{B} \rho$-determination depending which isotope range is selected. Far from the ideally
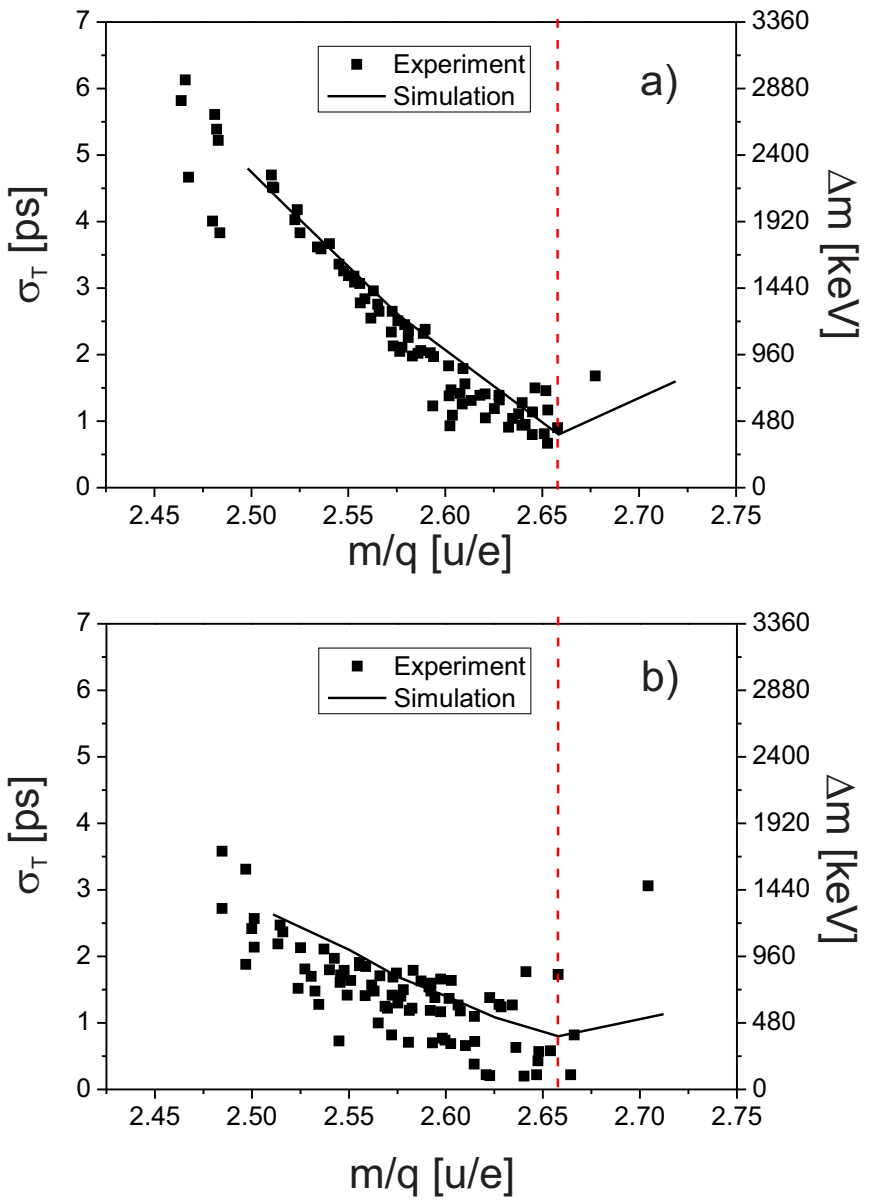

FIG. 6. Measured time resolution (standard deviation in ps) of stored ions for different $\mathrm{m} / \mathrm{q}$ values without (a) and with (b) $\mathrm{B} \rho$-tagging. The corresponding simulated time resolution calculated with MOCADI [26] is included by full lines. The parameters of the accepted $\mathrm{B} \rho$ range has been adjusted to match the experimental results.

isochronous region the factor is even larger. We have benefited for the particle identification and the observation of isomers from this improved resolution. A broader overview is presented in figure 6. As discussed in the previous section, it is advantageous that the measured ToF spectrum simultaneously contains nuclides with unknown and those with well-known masses, thus the calibrants experience the same possible drifts in the measurement cycles as the precious isotopes with unknown masses. Nevertheless, due to the limited resolving power (with respect to the standard deviation) of $(40-270) \cdot 10^{3}$ some close-lying peaks in the ToF spectrum could not be unambiguously identified and thus have been excluded from the present data analysis. In total, about 700 different peaks were unambiguously identified in the accumulated ToF spectrum and were analyzed. 


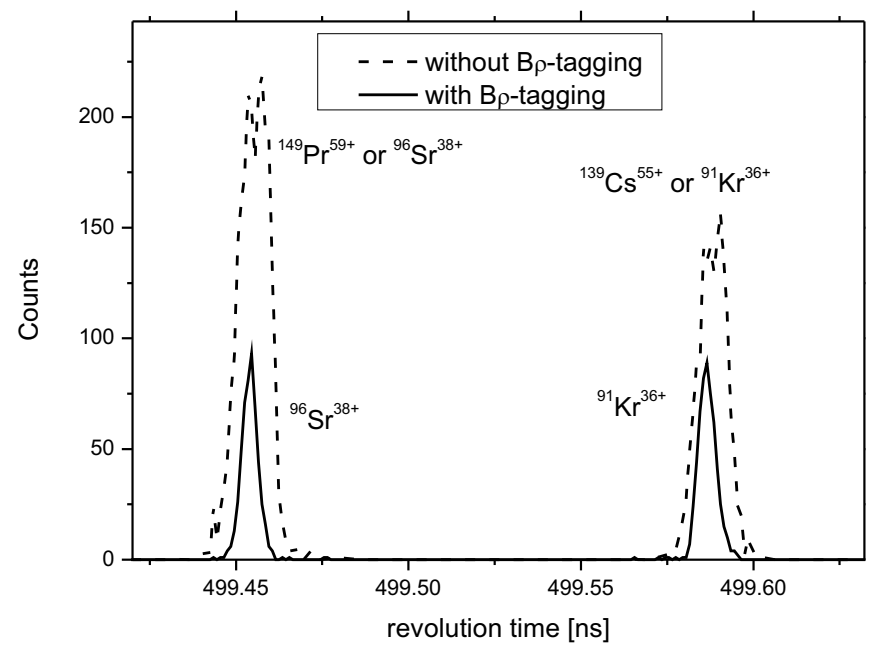

FIG. 7. Measured ToF spectra for different ions with and without $\mathrm{B} \rho$-tagging. The spectra without $\mathrm{B} \rho$-tagging are more than a factor of 2 broader. The unique particle identification can be only achieved in this case via $\mathrm{B} \rho$-tagging as demonstrated in the depicted examples

- Note that the measurement time for the two types of spectra were different. Therefore, the difference of the abundance does not reflect the transmission ratio.

\section{Matrix method}

The correlation matrix method, first developed for the analysis of Schottky mass measurements [19], was adapted and used to determine the mass values from the measured revolution times. The present evaluation has been modified compared with previous IMS data analysis. The new aspects are: i) the method was applied simultaneously to the data of the different experiments with and without $\mathrm{B} \rho$-tagging, ii) an additional $\mathrm{m} / \mathrm{q}$-correlation was included to take into account the non-isochronous region. iii) a correlation of the measured number of revolutions in the ring and the systematic error was applied. The matrix method is based on a maximum likelihood method to determine masses using the relation between measured unknown masses and reference masses. For each revolution time $T_{j}^{\mu}$ of an ion $\mu$ in a spectrum $j$ the measured $m / q$ value was approximated by a polynomial function with the coefficients $a_{j}^{k}$ and the order $k$

$$
m / q=\sum_{k=0} a_{j}^{k}\left(T_{j}^{\mu}\right)^{k}
$$

The measured standard deviation of the revolution time $\sigma_{T_{j}^{\mu}}$ was converted into the corresponding massover-charge uncertainty by:

$$
\Delta_{j}^{\mu}=s \sigma_{T_{j}^{\mu}}
$$

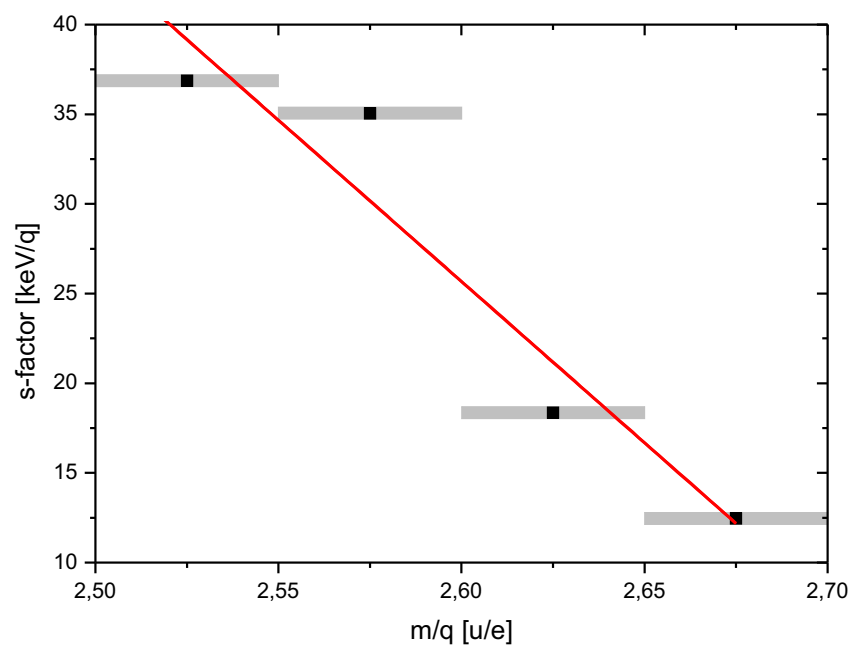

FIG. 8. Measured dependence of the s-factor for different $\mathrm{m} / \mathrm{q}$ values of the combined experiments. The horizontal bars represent $\mathrm{m} / \mathrm{q}$ ranges where the $\mathrm{s}$ factor is approximately constant. The black curve is a linear fit to the data. Its slope is in agreement with the $\mathrm{m} / \mathrm{q}$-dependence shown in figure 6 for the values smaller than the isochronous particle.

The uncertainty of the revolution-time for nonisochronous isotopes is systematically larger than for the isochronous ions. Therefore, a variable factor (s), dependent on the $m / q$-values, was implemented in this analysis for the first time, see figure 8 . As it can be seen from figures 6 and 8 , a proportionality is obtained between the mass resolution and the statistical uncertainty of the mass measurement. Such a linear dependence exists for most mass measurement techniques and confirms the necessity to establish a mass-over-charge dependent s-factor.

\section{Error contributions}

Ions with unknown masses and reference ions with well-known masses were simultaneously measured in the same spectrum. The mass values are calculated with the matrix method by

$$
\vec{M}=\boldsymbol{W}^{-1} \vec{w}
$$

The matrix elements of $\mathrm{W}$ are functions of the revolution time weighted with the uncertainty of each revolution time $\sigma_{T}$ and of the calibration coefficients from the 3rd order polynomial fit.

The statistical error is the diagonal element of the inverse matrix $\boldsymbol{W}^{-1}$ :

$$
\left(\sigma_{m_{\mu}}^{\text {stat }}\right)^{2}=\boldsymbol{W}_{\mu \mu}^{-1} .
$$




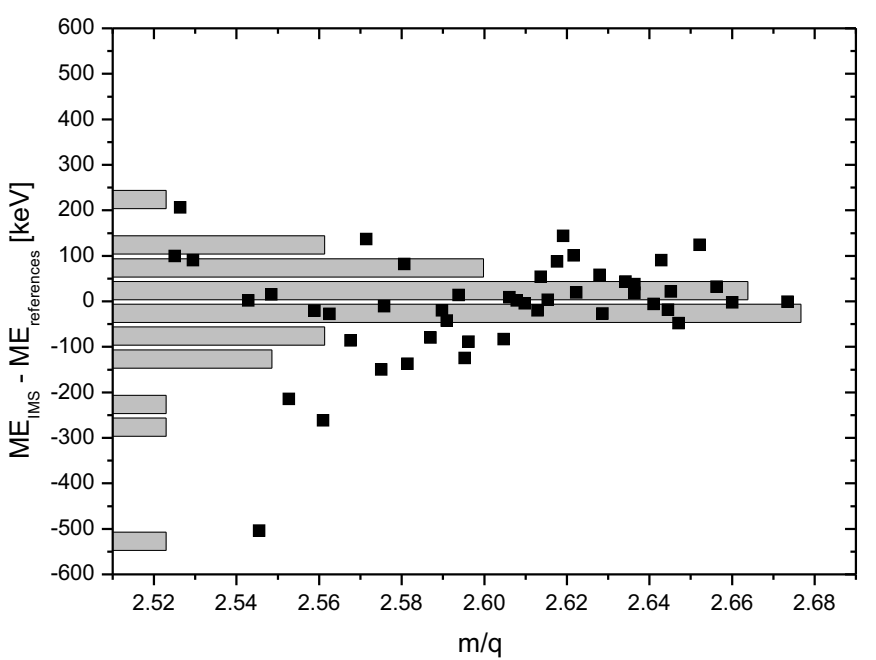

FIG. 9. The systematic error of our mass measurement deduced from re-analysis of each well-known reference mass, i.e., each reference mass is treated sequentially as an unknown species. The distribution of this analysis is depicted as a function of mass-over-charge values and shown in the insert as a projected histogram.

This error includes the uncertainties from the reference nuclei as well as the errors in the frequency determination. The statistical errors range from a few tens of $\mathrm{keV}$ up to several hundred keV for very rare ions measured in these experiments.

The reliability of the new data analysis has been verified by the determination of the systematic error of the combined experiments. In this analysis with the combined data sets, with and without $B \rho$-tagging, an average of 1600 events per reference mass were included. We determined the systematic error by using all reference masses and sequentially treated each of these masses as being unknown. The procedure can be illustrated by equation 6 and figure 9 .

$$
\sum_{i}^{n} \frac{\left(M_{i}-M_{i}^{r e f}\right)^{2}}{\left(\sigma_{i}^{r e f}\right)^{2}+\left(\sigma_{i}^{s t a t}\right)^{2}+\left(\sigma^{s y s t}\right)^{2}}=n,
$$

where $M_{i}^{r e f}$ are the mass values and $\sigma_{i}^{r e f}$ the uncertainties of the reference nuclides. $\sigma_{i}^{\text {stat }}$ are the statistical errors of the measured masses $M_{i} . n$ is the number of reference masses and $\sigma^{\text {syst }}$ the systematic error. In this analysis all accurately measured masses [28] were used to cover most of the $\mathrm{m} / \mathrm{q}$ range of the present analysis. The investigation yields a mean deviation of the projected distribution from the literature values of $1.29 \mathrm{keV}$ and a standard deviation of $172 \mathrm{keV}$. The latter represents a common systematic error $\left(\sigma^{\text {syst }}\right)$ for all new IMS results in this article. For the described procedure a Gaussian statistical distribution is assumed. However, for very rare nuclides characterized with a few recorded events in the whole experiment, a Gaussian description is very likely to underestimate the uncertainty. Indeed, we observed in detailed investigations a strong correlation between the mean measured uncertainty of the revolution time $\sigma_{T}$ and the number of turns recorded in the ring. The measured correlation is plotted in figure 10. An additional systematic error as a function of the reached maximum turn number has been taken into account. This additional error $\sigma^{\text {syst-turn }}$ is only significant for isotopes with less than 14 recorded ions and an average maximum turn number below 250. This contributes quadratically to the total error. For larger numbers of recorded ions this additional systematic error contribution is negligible. The experimental reason for this observed correlation is probably the influence of the initial phase-space coordinates, e.g., the velocity, position and angular coordinates of the injected ions. The transformation from the time uncertainty to the corresponding mass uncertainty was done by using the relation:

$$
\delta m=\delta T / T \cdot \gamma_{t}^{2} \cdot m
$$

This relation yields, for example, $480 \mathrm{keV} / \mathrm{ps}$ for an ion with a mass of $130 \mathrm{u}$ and an ionic charge of 50 . Hence the overall uncertainty of the measured mass excess is

$$
\Delta M E=\sqrt{\left(\sigma^{\text {stat }}\right)^{2}+\left(\sigma^{\text {syst-total }}\right)^{2}}
$$

where

$$
\sigma^{\text {syst-total }}=\sqrt{\left(\sigma^{\text {syst-turn }}\right)^{2}+\left(\sigma^{\text {syst }}\right)^{2}} .
$$

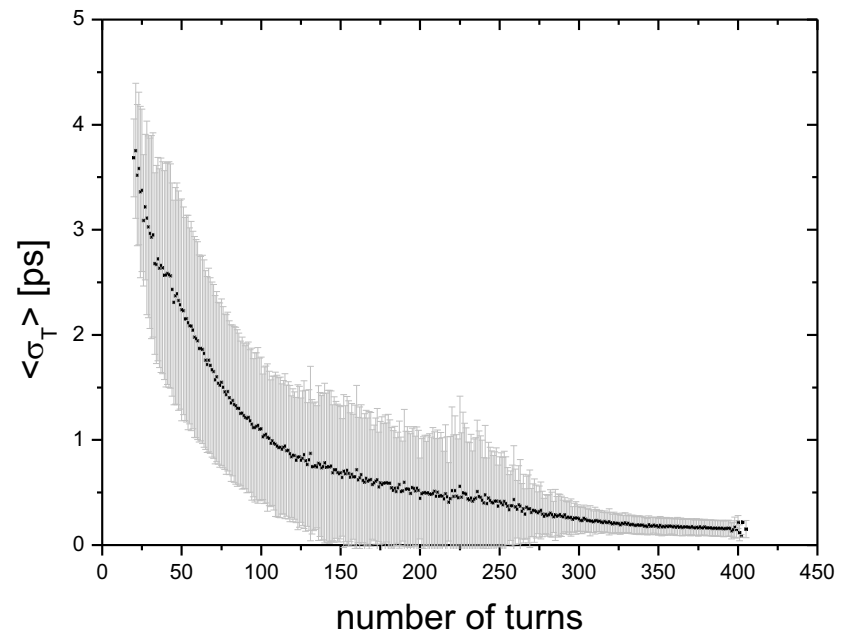

FIG. 10. Measured correlation between the spread of the revolution time and the number of turns recorded in the ring. The band around the mean values reflects the fluctuations which become small after about 250 turns.

The systematic error is the dominant contribution to the total error for most of the new mass values. How- 
ever, for the very rare nuclides with $\leq 14$ the additional correlation has a large impact on the total error.

\section{RESULTS OF MASS MEASUREMENTS}

The masses in the present experiments have been determined by accurate revolution-time measurements of reference nuclides with well-known masses combined with the ions for which we measured the mass values for the first time. Therefore, it is valuable not only to present here the new experimental data but also the reference masses used. In table I we list the reference masses applied for calibration of the experimental spectra. The mass-excess values ME, the corresponding accuracies $\triangle \mathrm{ME}$ and the deviation from the re-evaluated mass value $\left(M E-M E_{l i t}\right)$ applied in the matrix analysis are tabulated. In addition, the error $\Delta\left(M E-M E_{l i t}\right)$ for the difference $M E-M E_{\text {lit }}$ is presented in the 5th column. Furthermore, we state the total number of ions (counts) for each isotope recorded in the ESR.

TABLE I: List of reference masses used for calibration. The mass-excess values from the literature $\mathrm{ME}_{\text {lit }}$ and the corresponding accuracies $\Delta \mathrm{ME}_{l i t}$ are given [28]. In addition, the reevaluated values from the overall matrix-calculation are shown as differences ME-ME $\mathrm{E}_{l i t}$. Furthermore, we state the total number of recorded ions for each isotope for both experiments in the ESR.

\begin{tabular}{|c|c|c|c|c|c|}
\hline isotope & \begin{tabular}{|l|}
$\mathrm{ME}_{\text {lit }}$ \\
{$[\mathrm{keV}]$} \\
\end{tabular} & $\begin{array}{c}\Delta \mathrm{ME}_{l i t} \\
{[\mathrm{keV}]}\end{array}$ & $\begin{array}{c}{\mathrm{ME}-\mathrm{ME}_{\text {lit }}}_{[\mathrm{keV}]} \\
\end{array}$ & $\begin{array}{c}\Delta\left(\mathrm{ME}^{\mathrm{MEE}} \mathrm{ME}_{l i t}\right) \\
{[\mathrm{keV}]}\end{array}$ & counts \\
\hline${ }^{79} \mathrm{Ga}$ & -62547 & 1 & 15 & 172 & 706 \\
\hline${ }^{80} \mathrm{Ga}$ & -59223 & 2 & 82 & 172 & 358 \\
\hline${ }^{81} \mathrm{Ga}$ & -57628 & 3 & -20 & 172 & 138 \\
\hline${ }^{82} \mathrm{Ga}$ & -52930 & 2 & 22 & 172 & 47 \\
\hline${ }^{82} \mathrm{Ge}$ & -65415 & 2 & -27 & 172 & 1181 \\
\hline${ }^{83} \mathrm{Ge}$ & -60976 & 2 & 14 & 172 & 452 \\
\hline${ }^{85} \mathrm{Ge}$ & -53123 & 3 & 32 & 172 & 55 \\
\hline${ }^{85} \mathrm{As}$ & -63189 & 3 & -11 & 172 & 1204 \\
\hline${ }^{86} \mathrm{As}$ & -58962 & 3 & 9 & 172 & 568 \\
\hline${ }^{87} \mathrm{As}$ & -55617 & 3 & 19 & 172 & 116 \\
\hline${ }^{86} \mathrm{Se}$ & -70503 & 2 & 91 & 172 & 4381 \\
\hline${ }^{87} \mathrm{Se}$ & -66426 & 2 & -20 & 172 & 4174 \\
\hline${ }^{89} \mathrm{Se}$ & -58992 & 3 & 88 & 172 & 455 \\
\hline${ }^{89} \mathrm{Br}$ & -68275 & 4 & 2 & 172 & 8130 \\
\hline${ }^{90} \mathrm{Br}$ & -64001 & 4 & 137 & 172 & 3350 \\
\hline${ }^{92} \mathrm{Br}$ & -56233 & 7 & -27 & 173 & 164 \\
\hline${ }^{95} \mathrm{Rb}$ & -65935 & 4 & -86 & 172 & 7529 \\
\hline${ }^{97} \mathrm{Rb}$ & -58519 & 6 & 101 & 173 & 828 \\
\hline${ }^{96} \mathrm{Sr}$ & -72926 & 10 & 206 & 173 & 8976 \\
\hline${ }^{97} \mathrm{Sr}$ & -68587 & 10 & -214 & 173 & 7906 \\
\hline${ }^{101} \mathrm{Y}$ & -65065 & 8 & -20 & 173 & 4049 \\
\hline${ }^{103} \mathrm{Y}$ & -58457 & 11 & -6 & 173 & 59 \\
\hline${ }^{101} \mathrm{Zr}$ & -73164 & 10 & 100 & 173 & 7689 \\
\hline${ }^{103} \mathrm{Zr}$ & -67819 & 10 & -149 & 173 & 4079 \\
\hline${ }^{105} \mathrm{Nb}$ & -69907 & 5 & -262 & 173 & 405 \\
\hline${ }^{107} \mathrm{Nb}$ & -63715 & 9 & -4 & 173 & 1231 \\
\hline${ }^{108} \mathrm{Nb}$ & -59545 & 9 & 44 & 173 & 390 \\
\hline${ }^{109} \mathrm{Mo}$ & -66670 & 12 & -124 & 173 & 1390 \\
\hline${ }^{110} \mathrm{Mo}$ & |-64547 & 24 & 144 & 174 & 172 \\
\hline
\end{tabular}

\begin{tabular}{|c|c|c|c|c|c|}
\hline isotope & $\begin{array}{c}\mathrm{ME}_{\text {lit }} \\
{[\mathrm{keV}]}\end{array}$ & $\begin{array}{c}\Delta \mathrm{ME}_{\text {lit }} \\
{[\mathrm{keV}]}\end{array}$ & $\begin{array}{c}\mathrm{ME}-\mathrm{ME}_{\text {lit }} \\
{[\mathrm{keV}]}\end{array}$ & $\begin{array}{c}\Delta\left(\mathrm{ME}-\mathrm{ME}_{\text {lit }}\right) \\
{[\mathrm{keV}]}\end{array}$ & counts \\
\hline${ }^{111} \mathrm{Mo}$ & -59933 & 13 & 90 & 173 & 95 \\
${ }^{111} \mathrm{Tc}$ & -69018 & 11 & -137 & 173 & 156 \\
${ }^{112} \mathrm{Tc}$ & -65250 & 6 & -83 & 173 & 886 \\
${ }^{113} \mathrm{Tc}$ & -62812 & 4 & 58 & 173 & 368 \\
${ }^{112} \mathrm{Ru}$ & -75624 & 10 & -504 & 173 & 1284 \\
${ }^{114} \mathrm{Ru}$ & -70221 & 4 & -42 & 173 & 36 \\
${ }^{115} \mathrm{Ru}$ & -66071 & 8 & 54 & 173 & 580 \\
${ }^{116} \mathrm{Ru}$ & -64069 & 4 & 38 & 172 & 158 \\
${ }^{118} \mathrm{Rh}$ & -64894 & 24 & 20 & 174 & 331 \\
${ }^{119} \mathrm{Rh}$ & -62823 & 10 & -18 & 173 & 118 \\
${ }^{119} \mathrm{Pd}$ & -71415 & 9 & -79 & 173 & 55 \\
${ }^{122} \mathrm{Pd}$ & -64616 & 19 & 125 & 174 & 43 \\
${ }^{131} \mathrm{In}$ & -68025 & 2 & 0 & 172 & 19 \\
${ }^{133} \mathrm{Sn}$ & -70874 & 2 & -2 & 172 & 184 \\
${ }^{133} \mathrm{Sb}$ & -78921 & 4 & 2 & 173 & 128 \\
${ }^{135} \mathrm{Sb}$ & -69689 & 2 & -48 & 172 & 1892 \\
${ }^{135} \mathrm{Te}$ & -77727 & 2 & -88 & 172 & 2606 \\
${ }^{136} \mathrm{Te}$ & -74425 & 2 & 3 & 172 & 14 \\
\hline
\end{tabular}

The masses measured for the first time and those which were improved in accuracy are presented with the corresponding statistical and systematic errors in the tables II and III, respectively. The masses measured in the present experiments for the first time are compared with the extrapolated values of reference [29] in figure 11 . The deviations observed in this comparison are up to $1.6 \mathrm{MeV}$. In general, there is a slight tendency observed that the extrapolated mass values have lower mass-excess values. The mean value of the projection is about $-200 \mathrm{keV}$. In figure 12 our improved mass values are compared with the compilation AME2012. The deviation of the mass values from the AME2012 is a factor of two less compared to figure 11, and most of the values are within the experimental uncertainties.

TABLE II: List of mass excess values measured for the first time. The $\triangle \mathrm{ME}$ values represent the total experimental errors, which consist of the contributions from the total systematic errors $\sigma^{\text {syst-total }}$ and the statistical errors $\sigma^{\text {stat }}$. The last column indicates the number of events recorded in the experiments.

\begin{tabular}{|c|c|c|c|c|c|}
\hline isotope & $\begin{array}{c}\mathrm{ME} \\
{[\mathrm{keV}]}\end{array}$ & $\begin{array}{c}\Delta \mathrm{ME} \\
{[\mathrm{keV}]}\end{array}$ & $\begin{array}{c}\sigma^{\text {syst-total }} \\
{[\mathrm{keV}]}\end{array}$ & $\begin{array}{c}\sigma^{\text {stat }} \\
{[\mathrm{keV}]}\end{array}$ & counts \\
\hline${ }^{86} \mathrm{Ge}$ & -49406 & 175 & 172 & 30 & 30 \\
${ }^{91} \mathrm{Se}$ & -50577 & 173 & 172 & 7 & 16 \\
${ }^{94} \mathrm{Br}$ & -46812 & 400 & 400 & 13 & 9 \\
${ }^{105} \mathrm{Y}$ & -51280 & 535 & 525 & 102 & 5 \\
${ }^{106} \mathrm{Zr}$ & -58547 & 173 & 172 & 6 & 120 \\
${ }^{107} \mathrm{Zr}$ & -54382 & 449 & 449 & 15 & 11 \\
${ }^{110} \mathrm{Nb}$ & -52315 & 335 & 335 & 14 & 3 \\
${ }^{113} \mathrm{Mo}$ & -53402 & 314 & 311 & 46 & 3 \\
${ }^{114} \mathrm{Tc}$ & -58595 & 173 & 172 & 8 & 61 \\
${ }^{115} \mathrm{Tc}$ & -56323 & 316 & 316 & 8 & 9 \\
${ }^{118} \mathrm{Ru}$ & -57633 & 183 & 181 & 26 & 2 \\
${ }^{121} \mathrm{Rh}$ & -56265 & 248 & 247 & 25 & 2 \\
${ }^{123} \mathrm{Pd}$ & -60428 & 316 & 315 & 29 & 10 \\
${ }^{124} \mathrm{Pd}$ & -60200 & 372 & 367 & 61 & 4 \\
${ }^{126} \mathrm{Ag}$ & -61411 & 306 & 306 & 17 & 14 \\
${ }^{138} \mathrm{Sb}$ & -54253 & 426 & 423 & 54 & 9 \\
\hline
\end{tabular}




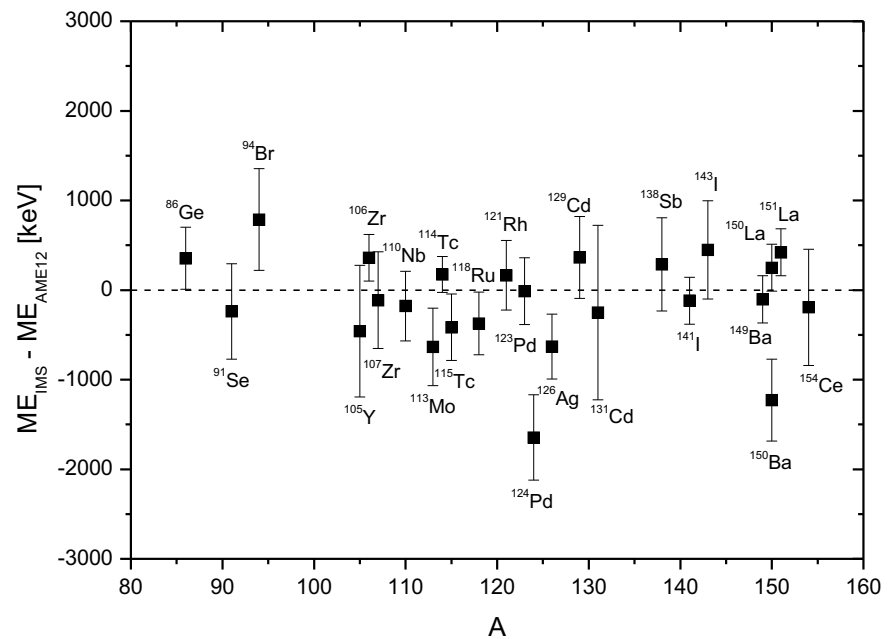

FIG. 11. Measured new mass values compared with the corresponding extrapolated AME2012 values.

\begin{tabular}{|c|c|c|c|c|c|}
\hline isotope & $\begin{array}{c}\mathrm{ME} \\
{[\mathrm{keV}]}\end{array}$ & $\begin{array}{c}\Delta \mathrm{ME} \\
{[\mathrm{keV}]}\end{array}$ & $\begin{array}{c}\sigma^{\text {syst-total }} \\
{[\mathrm{keV}]}\end{array}$ & $\begin{array}{c}\sigma^{\text {stat }} \\
{[\mathrm{keV}]}\end{array}$ & counts \\
\hline \hline${ }^{141} \mathrm{I}$ & -60023 & 173 & 172 & 7 & 177 \\
${ }^{143} \mathrm{I}$ & -50178 & 461 & 458 & 46 & 6 \\
${ }^{149} \mathrm{Ba}$ & -53124 & 175 & 172 & 29 & 29 \\
${ }^{150} \mathrm{Ba}$ & -51482 & 346 & 317 & 140 & 3 \\
${ }^{150} \mathrm{La}$ & -56135 & 174 & 172 & 24 & 26 \\
${ }^{151} \mathrm{La}$ & -53308 & 174 & 172 & 22 & 47 \\
${ }^{154} \mathrm{Ce}$ & -52543 & 577 & 573 & 67 & 6 \\
\hline
\end{tabular}

TABLE III: List of improved mass excess values. The $\triangle \mathrm{ME}$ values represent the total experimental errors, which consist of the contributions from the total systematic errors $\sigma_{\text {syst-total }}$ and the statistical errors $\sigma_{\text {stat }}$. The last column indicates the number of events recorded in the experiments.

\begin{tabular}{|c|c|c|c|c|c|}
\hline isotope & $\begin{array}{c}\mathrm{ME} \\
{[\mathrm{keV}]}\end{array}$ & $\begin{array}{c}\Delta \mathrm{ME} \\
{[\mathrm{keV}]}\end{array}$ & $\begin{array}{c}\sigma^{\text {syst-total }} \\
{[\mathrm{keV}]}\end{array}$ & $\begin{array}{c}\sigma^{\text {stat }} \\
{[\mathrm{keV}]}\end{array}$ & counts \\
\hline \hline${ }^{93} \mathrm{Br}$ & -52884 & 172 & 172 & 4 & 79 \\
${ }^{109} \mathrm{Nb}$ & -56690 & 172 & 172 & 4 & 76 \\
${ }^{117} \mathrm{Ru}$ & -59493 & 173 & 172 & 5 & 33 \\
${ }^{125} \mathrm{Ag}$ & -64514 & 173 & 172 & 6 & 66 \\
${ }^{137} \mathrm{Sb}$ & -60620 & 173 & 172 & 15 & 94 \\
${ }^{140} \mathrm{I}$ & -63529 & 173 & 172 & 5 & 750 \\
\hline
\end{tabular}

Results of new mass measurements are an important test bench for the predictive power of theoretical models. In figure 13 we give a representative comparison of our new and improved mass values for iodine and zirconium isotopes with different mass models. The models are based on microscopic-macroscopic descriptions [3, 33, 34], the Hartree-Fock-Bogoliubov (HFB) theory [30], and the shell-model inspired model of Duflo-Zuker [31]. It is clearly seen that the deviation becomes largest for the most neutron-rich ions, i.e. the previously experimentally unknown masses. In general, it is observed that the HFB-27 has a remarkably good predictive power in the range of our new mass values.

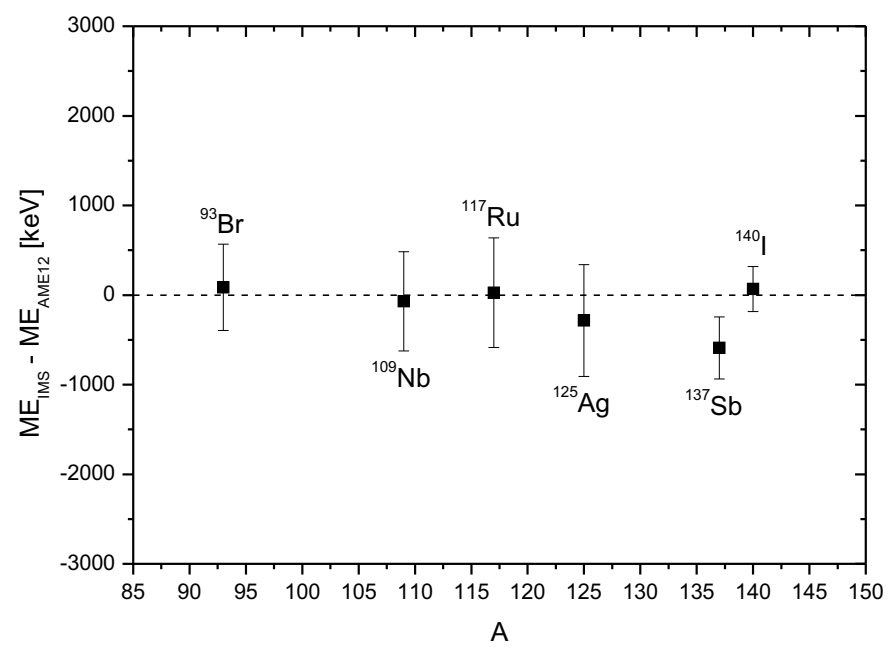

FIG. 12. Measured mass values with improved uncertainties compared with the corresponding AME2012 values.
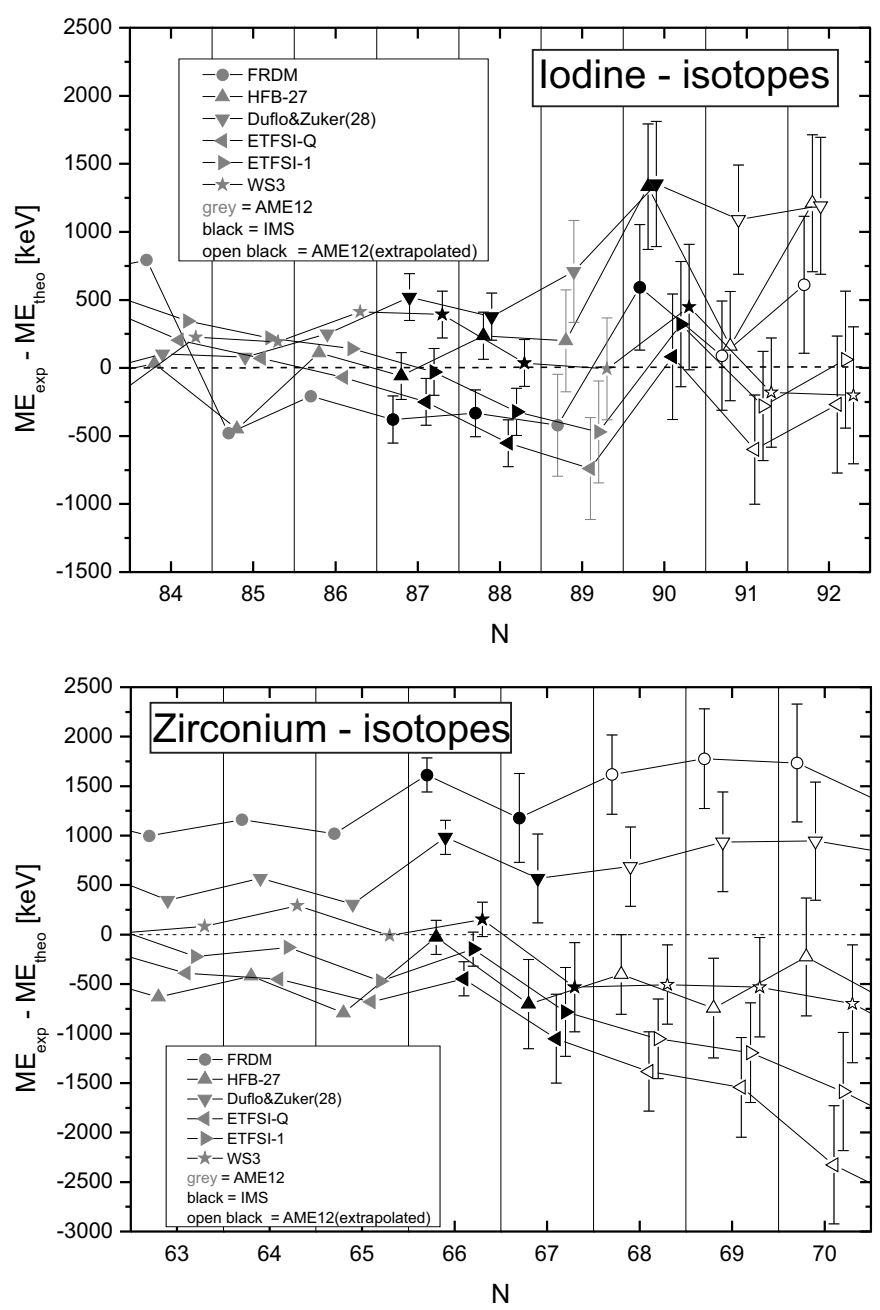

FIG. 13. The measured mass values for iodine (upper panel) and for zirconium (lower panel) isotopes are compared with the predictions of different theories. 
TABLE IV. Comparison of measured mass values with different models. RMS deviations for I and $\mathrm{Zr}$ isotopes for different theoretical models are presented. In this comparison the new IMS values and the tabulated experimental and extrapolated values of AME2012 are included. The columns $\sigma_{r m s, I}$, $\sigma_{r m s, Z r}$ represent the rms-values for I and the $\mathrm{Zr}$ isotopes in the mass range as shown in figure 13 .

\begin{tabular}{|c|c|c|}
\hline & $\begin{array}{c}\sigma_{r m s, I} \\
{[\mathrm{keV}]}\end{array}$ & $\begin{array}{c}\sigma_{r m s, Z r} \\
{[\mathrm{keV}]}\end{array}$ \\
\hline FRDM [3] & 479 & 1421 \\
HFB-27 [30] & 631 & 555 \\
DZ28 [31] & 777 & 713 \\
ETFSI-Q [32] & 395 & 1216 \\
ETFSI-1 [33] & 279 & 864 \\
WS3 [34] & 276 & 422 \\
\hline
\end{tabular}

TABLE V. Comparison of the new mass values from Table II and the one-neutron separation energies $\left(S_{n}\right.$ values) with different models. RMS deviations are calculated for the different isotopes and also the shift of the mean values. In the comparison of the $\mathrm{S}_{n}$ values the tabulated experimental and extrapolated values of AME2012 are included.

\begin{tabular}{|c|c|c|c|}
\hline & $\begin{array}{c}\sigma_{r m s, \mathrm{ME}} \\
{[\mathrm{keV}]}\end{array}$ & $\begin{array}{c}\left\langle\mathrm{ME}_{I M S}-\mathrm{ME}_{\text {theory }}\right\rangle \\
{[\mathrm{keV}]}\end{array}$ & $\begin{array}{c}\sigma_{r m s, S_{n}} \\
{[\mathrm{keV}]}\end{array}$ \\
\hline AME2012 [29] & 558 & -119 & 558 \\
FRDM [3] & 797 & 461 & 724 \\
HFB-27 [30] & 637 & 129 & 744 \\
DZ28 [31] & 855 & 558 & 787 \\
ETFSI-Q [32] & 1080 & -723 & 708 \\
ETFSI-1 [33] & 812 & -350 & 655 \\
WS3 [34] & 654 & 48 & 852 \\
\hline
\end{tabular}

The predictive power of the different models can be quantitatively characterized by the $\sigma_{r m s}$ values:

$$
\sigma_{r m s}=\sqrt{\frac{1}{n} \sum_{i=1}^{n}\left(M E-M E_{\text {theory }}\right)_{i}{ }^{2}} .
$$

They are listed in table IV for iodine and zirconium isotopes.

The one-neutron separation energy $S_{n}$ is defined by:

$$
S_{n}=-M(Z, N)+M(Z, N-1)+M(0,1),
$$

where $\mathrm{A}$ and $\mathrm{Z}$ are the mass and proton numbers, respectively. It is interesting also to compare the one-neutron separation energies $\left(S_{n}\right)$ for the new masses with the same theoretical models used for the comparison of the mass excess values. In some regions of the mass surface the deviations of the experimental and theoretical
$S_{n}$ values are systematically smaller than the corresponding comparison with the direct mass or ME values, because deficiencies of models can cancel in the subtraction. The results of the comparison of the $S_{n}$ values are summarized in the calculated rms values in table $\mathrm{V}$. In this comparison we observe the expected trend except for the Hartree-Fock-Bogoliubov (HFB) theory [30] and the model of reference [34].

\section{SUMMARY AND PERSPECTIVES}

IMS experiments have been performed with and without $\mathrm{B} \rho$-tagging with the FRS-ESR facilities at GSI. In the experiment with $\mathrm{B} \rho$-tagging the high ion-optical resolution of the lateral dispersive central focal plane of the FRS was used to determine the velocity of the different ions injected into the storage ring ESR. 25 new mass values in the range of $\mathrm{Ge}$ to $\mathrm{Ce}(\mathrm{A}=86-154)$ were obtained with a new method of data analysis, using the correlation matrix for the combined data of both types of experiments. The new masses of the isotopes ${ }^{129,130,131} \mathrm{Cd}$ have been published separately in reference [4]. Reliable mass values were obtained even for isotopes measured with a few atoms per week. The latter condition clearly demonstrates the high sensitivity and selectivity of the experimental method.

In this article we have presented in detail the new data analysis including the involved error evaluation. A proportionality between the mass resolution and the statistical uncertainty of the mass measurement has been observed. Therefore, an $\mathrm{m} / \mathrm{q}$ dependent scaling factor (s) in the analysis is necessary.

For the ions which were observed with a few counts only during the experiments we have an additional systematic error included based on a new correlation observed for the time uncertainty and the number of turns an ion has been recorded in the ESR. The systematic errors are dominating in most cases. The new and improved mass values have been listed in tables and compared to mass models.

In the next-generation IMS experiments we will improve the isochronous conditions in the storage ring and will measure the $\mathrm{B} \rho$ or velocity inside of the ring in addition to the revolution frequency. Measurements of smaller $\mathrm{m} / \mathrm{q}$ windows centred around the selected isochronous pilot fragment will also reduce the uncertainties due to imperfections of the isochronisity. From this experience one practical conclusion is that we will install two ToF detectors in the storage ring to measure the mean velocity and the revolution frequency simultaneously as proposed in reference [17]. These new technical developments are described in [35]. 


\section{ACKNOWLEDGEMENTS}

This work was supported by the German Federal Ministry of Education and Research (BMBF) numbers 06GI9115I and 06DA7047I, the Helmholtz Association (HGF) through the Nuclear Astrophysics Virtual Institute (VH-VI-417), the Helmholtz International Center for FAIR (HIC for FAIR) within the framework of the LOEWE program launched by the State of Hesse, and the UK Science and Technology Facilities Council.

[1] A. Bohr and B. Mottelson, Nuclear Structure, Vol. I (World Scientific, 1998).

[2] F.-K. Thielemann et al., Nucl. Phys. A 751, 301 (2005).

[3] P. Möller et al., At. Data Nucl. Data Tables 59, 185 (1995).

[4] R. Knöbel et al., Phys. Lett. B (2016), in print.

[5] H. Geissel et al., eds., Encyclopedia of Nuclear Physics and its Applications (Wiley-VCH, 2013).

[6] Y. A. Litvinov and K. Blaum, eds., "100 years of Mass Spetrometry," (Int. J. Mass Spectrom., 2013) pp. 1-276.

[7] T. Kubo et al., Nucl. Instr. and Meth. in Phys. Res. B 204 (2003).

[8] J. Kurcewicz et al., Phys. Lett. B 717, 371375 (2012).

[9] J. Dobaczewski et al., Phys. Rev. Lett. 72, 981 (1994).

[10] T. Otsuka et al., Phys. Rev. Lett. 87 (2001).

[11] C. Thibault et al., Phys. Rev. C 12, 644 (1975).

[12] A. Ozawa et al., Phys. Rev. Lett. 84, 5493 (2000).

[13] R. Kanungo, Phys. Scr. 2013 (2013).

[14] B. Franzke, H. Geissel, and G. Münzenberg, Mass Spectrom. Rev. 27, 428 (2008).
[15] W. R. Plaß, T. Dickel, and C. Scheidenberger, Int. J. Mass Spectrom. 349, 134 (2013).

[16] M. Hausmann et al., Nucl. Instrum. Meth. A 446, 569 (2000).

[17] H. Geissel et al., Hyperfine Interact. 173, 49 (2006).

[18] H. Geissel et al., Nucl. Instrum. Meth. B 70, 286 (1992).

[19] T. Radon et al., Nucl. Phys. A 677, 75 (2000).

[20] B. Franczak, in Europhysics Conference on Computing in Accelerator Design and Operation (Berlin, 1983).

[21] B. Franzke, Nucl. Instrum. Meth. B 24/25, 18 (1987).

[22] B. Blasche and B. Franczak, in Proceedings of European Particle Accelerator Conference, Vol. 9 (1992) p. 37.

[23] B. Sun, Ph.D. thesis, Beijing University, China (2008).

[24] H. Geissel et al., Microscopy and Microanalysis Microscopy and MIcroanalysis, 21, 160 (2015).

[25] J. Trötscher et al., Nucl. Instrum. Meth. B 70, 455 (1992).

[26] N. Iwasa, H. Weick, and H. Geissel, Nucl. Instr. Meth. B 269, 752 (2011).

[27] M. Diwisch, Ph.D. thesis, Justus-Liebig-University Giessen (2015).

[28] JYFLTRAP, http://research.jyu.fi/igisol/ JYFLTRAP_masses/nrich.html (2012), [Online; accessed 01-May-2015].

[29] M. Wang, G. Audi, and A. Wapstra, Chin. Phys. C 36, 1603 (2012).

[30] S. Goriely, N. Chamel, and J. Pearson, Phys. Rev. C 88, 024308 (2013).

[31] J. Duflo and A. Zuker, Phys. Rev. C 52, R23 (1995).

[32] J. Pearson et al., Phys. Lett. B 387, 455 (1996).

[33] Y. Aboussir et al., At. Data Nucl. Data Tables 61, 127 (1995).

[34] N. Wang and M. Liu, J. Phys. 420, 012057 (2013).

[35] M. Diwisch et al., Phys. Scr. T166, 014058 (2015). 